\title{
Reflectance confocal microscopy and dermoscopy for in vivo, non-invasive skin imaging of superficial basal cell carcinoma
}

\author{
MIHAELA A. GHITA ${ }^{1}$, CONSTANTIN CARUNTU ${ }^{2,3}$, ADRIAN E. ROSCA ${ }^{2,4}$, HARILLAQ KALESHI $^{3}$, \\ ANA CARUNTU $^{5}$, LILIANA MORARU ${ }^{5}$, ANCA OANA DOCEA $^{6}$, SABINA ZURAC $^{7}$, DANIEL BODA $^{1}$, \\ MONICA NEAGU ${ }^{4}$, DEMETRIOS A. SPANDIDOS ${ }^{8}$ and ARISTIDIS M. TSATSAKIS ${ }^{9}$
}

\begin{abstract}
${ }^{1}$ Dermatology Research Laboratory, and ${ }^{2}$ Department of Physiology, 'Carol Davila' University of Medicine and Pharmacy, Bucharest 050474; ${ }^{3}$ Department of Dermatology, 'Prof. N. Paulescu' National Institute of Diabetes, Nutrition and Metabolic Diseases, Bucharest 011233; ' ${ }^{4}$ Victor Babes' National Institute of Pathology, Bucharest 050096; ${ }^{5}$ Department of Oral and Maxillofacial Surgery, 'Carol Davila' Central Military Emergency Hospital, Bucharest 010825; ${ }^{6}$ Department of Toxicology, Faculty of Pharmacy, University of Medicine and Pharmacy, Craiova 200349; ${ }^{7}$ Department of Pathology, 'Carol Davila' University of Medicine and Pharmacy, Colentina University Hospital, Bucharest 020125, Romania; ${ }^{8}$ Laboratory of Clinical Virology,

University of Crete Medical School, Heraklion 71409; ${ }^{9}$ Department of Forensic Sciences and Toxicology,

Medical School, University of Crete, Heraklion 71003, Greece
\end{abstract}

Received January 29, 2016; Accepted March 18, 2016

DOI: $10.3892 / 01.2016 .4354$

\begin{abstract}
Superficial basal cell carcinoma (sBCC) is the second most frequent histological type of basal cell carcinoma (BCC), usually requiring a skin biopsy to confirm the diagnosis. It usually appears on the upper trunk and shoulders as erythematous and squamous lesions. Although it has a slow growth and seldom metastasizes, early diagnosis and management are of crucial importance in preventing local invasion and subsequent disfigurement. Dermoscopy is nowadays an indispensable tool for the dermatologist when evaluating skin tumors. Reflectance confocal microscopy (RCM) is a novel imaging technique that allows the non-invasive, in vivo quasi-microscopic morphological and dynamic assessment of superficial skin tumors. Moreover, it offers the advantage of performing infinite repeatable determinations to monitor disease progression and non-surgical treatment for sBCC. Herein, we present three lesions of sBCC evaluated using in vivo and non-invasive imaging techniques, emphasizing the usefulness of combining RCM with dermoscopy for increasing the diagnostic accuracy of $\mathrm{sBCC}$.
\end{abstract}

\section{Introduction}

Superficial basal cell carcinoma (sBCC) is the second most frequent type after nodular basal cell carcinoma (BCC), and it

Correspondence to: Dr Constantin Caruntu, Department of Physiology, 'Carol Davila' University of Medicine and Pharmacy, 8 Eroii Sanitari, Bucharest 050474, Romania

E-mail: costin.caruntu@gmail.com

Key words: superficial basal cell carcinoma, dermoscopy, reflectance confocal microscopy, non-invasive, in vivo skin imaging typically appears on the trunk, as erythematous and squamous lesions, that can be very difficult to differentiate from other skin diseases with a similar clinical presentation. Although it usually has a slow growth and seldom metastasizes, if left untreated, sBCC lesions can produce extensive local distruction, causing significant morbidity or disfigurement. Early diagnosis and management are of crucial importance in preventing detrimental outcomes (1-3).

Reflectance confocal microscopy (RCM) is a novel high resolution imaging technique that allows the rapid and reproducible evaluation of $\mathrm{SBCC}$ in combination with dermoscopy (4), avoiding the invasiveness and high costs associated with skin biopsies. In this study, we aimed to expound the clinical usefulness of combining RCM and dermoscopy for the in vivo, non-invasive diagnosis of sBCC. For this purpose, we present three lesions of $\mathrm{sBCC}$ which we evaluated using in vivo and non-invasive imaging techniques.

\section{Case reports}

Case 1. A 46-year-old man, phototype III [fair to matte skin, sometimes burns, always tans (medium tan) with a few freckles], presented in March 2014 to the Dermatology Department of 'Prof. N. Paulescu' National Institute of Diabetes, Nutrition and Metabolic Diseases in Bucharest for the evaluation of a slow-growing lesion on his back, which he had first noticed 2 years ago. He reported significant sun exposure during his lifetime, consistent with the mottled telangiectatic and lentiginous background skin observed upon clinical examination. On his left upper back, there was a pink-colored patch with telangiectasias and ill-defined borders, measuring approximately 2.1x1 cm (Fig. 1A). Dermoscopy [all dermoscopic images in our study were captured at 10 -fold magnification prior to RCM imaging using the integrated VivaScope 1500 VivaCam macro camera (Lucid Inc., Rochester, NY, USA)] 

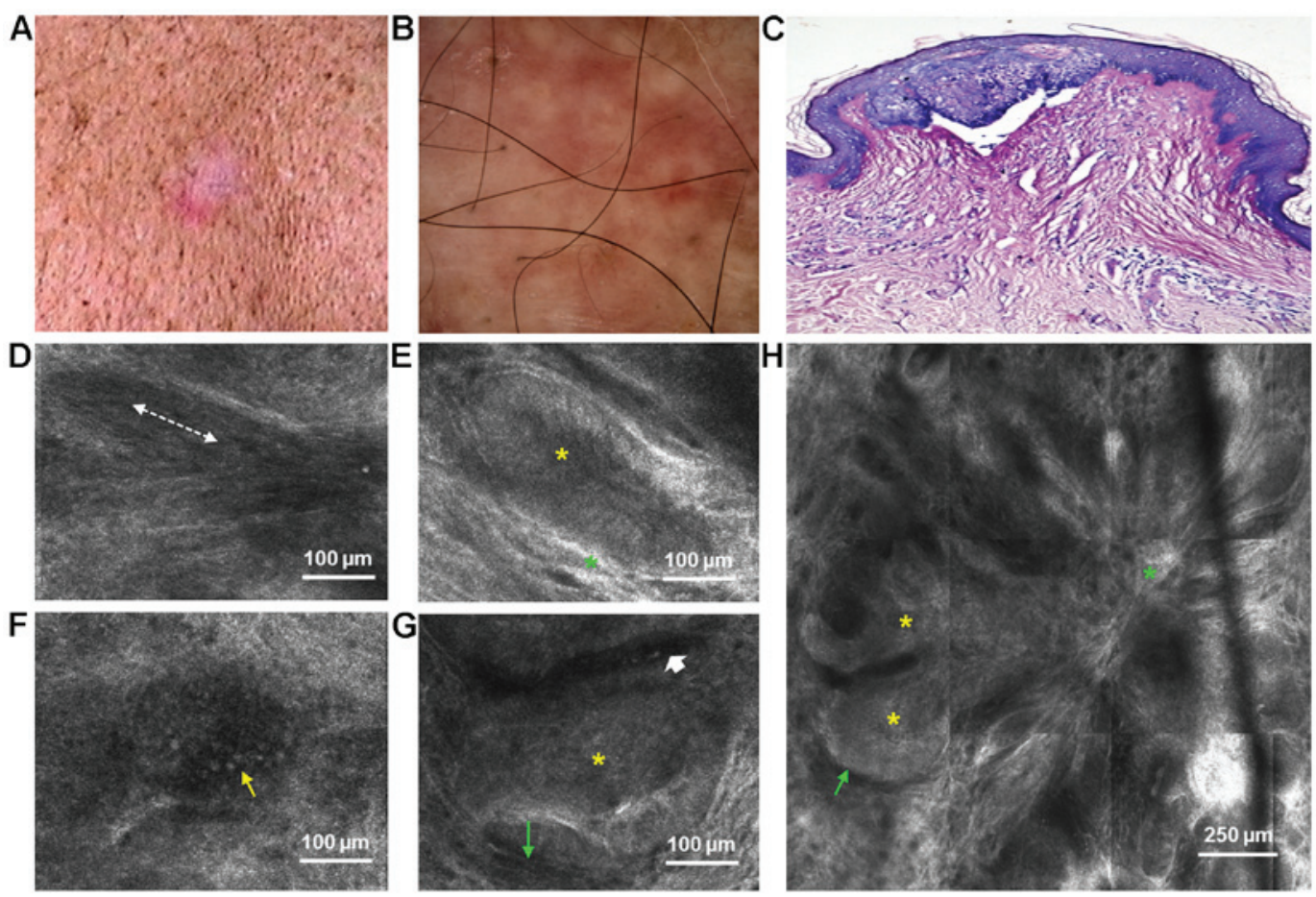

Figure 1. Lesion no. 1. (A) Clinical image showing a pink-colored patch with telangiectasias and ill-defined borders on a lentiginous background skin; (B) corresponding dermoscopic image with pink to red structureless areas, arborizing microvessels, short fine telangiectasias and few blue-gray globules; (C) histopathological image displaying a small island of basaloid cells with peripheral palisading that is connected in multiple places to the epidermis (Giemsa staining, x10 magnification); (D) Reflectance confocal microscopy (RCM) image at the level of the stratum spinosum showing streaming of the epidermis (dashed white line); (E) RCM image just below the epidermis showing an island of elongated tumor cells with polarized nuclei forming the typical peripheral palisade (yellow asterisk), surrounded by highly refractile collagen bundles (green asterisk); (F) RCM image in the upper dermis revealing an inflammatory cell infiltrate (thin yellow arrow); (G) RCM image of a typical island of basaloid cells with peripheral palisading (yellow asterisk) and thin peritumoral dark spaces (thin green arrow). A dilated, horizontal capillary can be seen adiacent to the tumor island (short white arrow); (H) RCM image revealing aggregations of basaloid cells (yellow asterisks), surrounded by peritumoral, dark spaces (thin green arrow), connected into a multilobular, flower-like architecture by highly-reflactile, fibrous tissue (green asterisk).

revealed pink to red structureless areas with arborizing microvessels, short fine telangiectasias and few blue-gray globules (Fig. 1B). RCM examination revealed the presence of multiple, small islands of basaloid cells, connected into a multilobular, flower-like architecture by highly-reflactile, fibrous tissue in the superficial dermis (Fig. 1H). Tumor islands exhibited peripheral palisading of nuclei and were surrounded by peritumoral, dark spaces (Fig. 1E and G). At the level of the spinous layer, keratinocytes with elongated nuclei, oriented along the same axis formed the typical 'streaming of the epidermis' (Fig. 1D). Numerous inflammatory cells (Fig. 1F) and dilated capillaries (Fig. 1G) were also observed. A histopathological examination confirmed the diagnosis of sBCC. It was characterized by small aggregates of basaloid cells with peripheral palisading and prominent clefting in the papillary dermis, connected to the base of the epidermis (Fig. 1C).

Case 2. A 64-year-old woman, also phototype III, without any significant personal and familial medical history, presented in February 2014 with 2 slow-growing lesions to the Dermatology Department of 'Prof. N. Paulescu' National Institute of Diabetes, Nutrition and Metabolic Diseases in Bucharest. The first lesion, located on her right forearm, was an erythematosquamous plaque with an irregular border, measuring approximately $2.5 \times 2.3 \mathrm{~cm}$ (Fig. 2A). Dermoscopy revealed pink-white structureless areas, small erosions, arborizing microvessels, superficial fine telangiectasias and brown dots (Fig. 2B). RCM of the superficial dermis identified tumor islands surrounded by peritumoral dark spaces (Fig. 2E), dark silhouettes (Fig. 2H), an erosion, increased vascularization (Fig. 2F) and bright inflammatory cells (Fig. 2G). Dark silhouettes correspond to the hypopigmented variant of tumor islands. At the level of the stratum spinosum, the presence of streaming of the epidermis was also observed (Fig. 2D). A histopathological analysis confirmed the diagnosis of sBCC. The main findings were represented by multiple small islands of basaloid cells with peripheral palisading that emanate from the undersurface of the epidermis into a loose fibromucinous stroma. Moreover, a small loss of substance covered by hematic and lymphocytic exudates could be observed within the tumor and an abundand peritumoral inflammatory infiltrate consisting of lymphocytes, histiocytes and rare melanophages (Fig. 2C).

The second lesion, located on the patient's left shoulder, was an erythematous, slightly scaly plaque with a stelate shape, measuring $2.5 \times 2.1 \mathrm{~cm}$. Telangiectasias could be observed on its surface and a brownish hue at its periphery (Fig. 3A). Dermoscopy revealed pink to red structureless areas, multiple arborizing microvessels, superficial fine telangiectasias and few brown dots (Fig. 3B). RCM revealed the presence of hyporefractile tumor islands surrounded by highly refractile fibrous stroma (Fig. 3F), tortuous, dilated capillaries with leukocyte trafficking (Fig. 3D and $\mathrm{H}$ ) and clustered inflammatory cells (Fig. 3G). Plump cells, described as large, bright, 


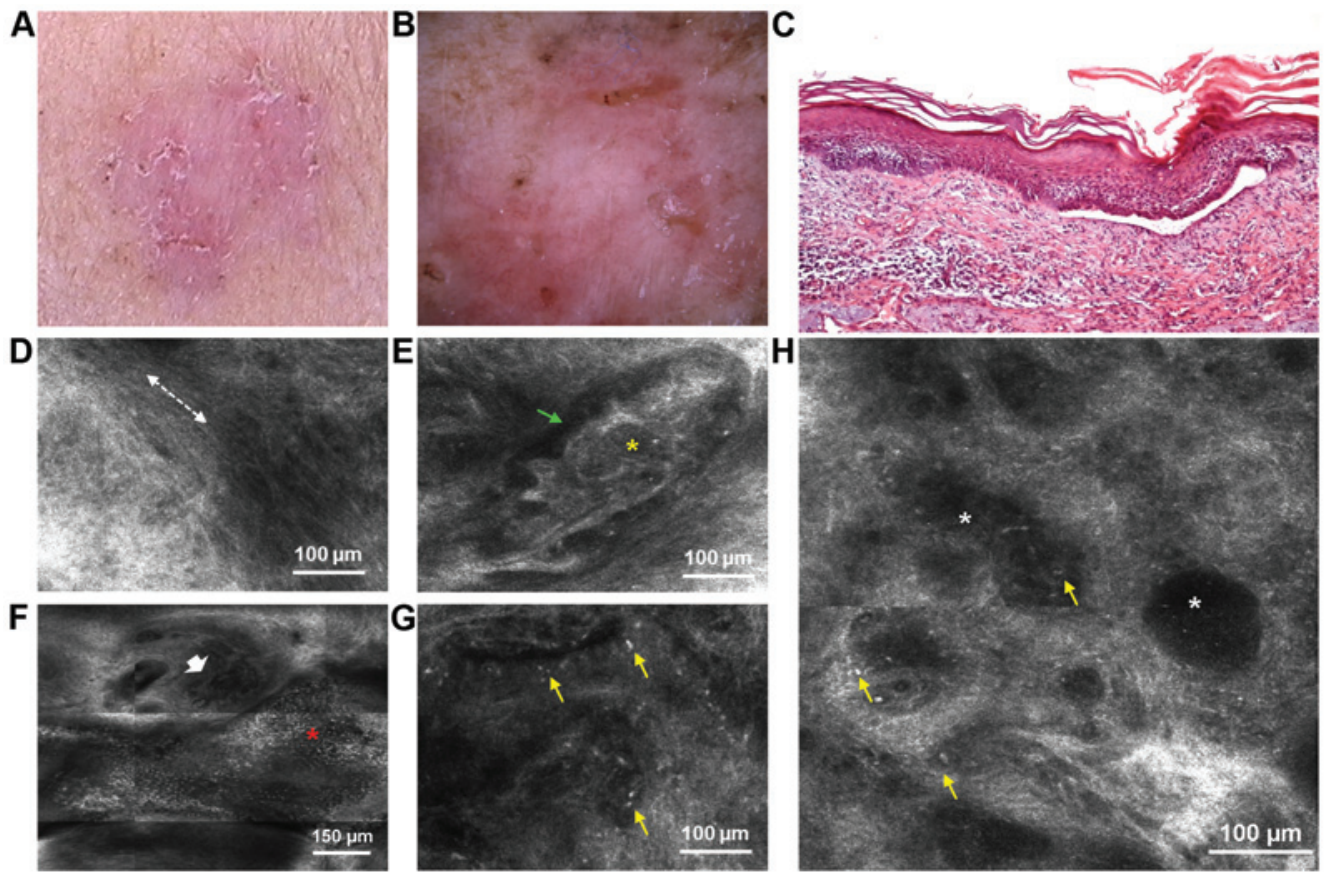

Figure 2. Lesion no. 2. (A) Clinical image of an erythematosquamous plaque with an irregular border; (B) corresponding dermoscopic appearance with pink-white structureless areas, small erosions, arborizing microvessels, superficial fine telangiectasias and brown dots; (C) Histopathological image displaying islands of basaloid cells with peripheral palisading that emanate from the undersurface of the epidermis (hematoxylin and eosin staining, x10 magnification); (D) Reflectance confocal microscopy (RCM) image at the level of the stratum spinosum showing streaming of the epidermis (dashed white line); (E) RCM image revealing a lobulated tumor island (yellow asterisk) surrounded by peritumoral dark spaces (thin green arrow); (F) RCM image showing an erosion (red asterisk) and at its superior pole, entwined capillaries (short white arrow); (G) RCM image of bright inflammatory cells (thin yellow arrows); (H) RCM image at the level of upper dermis showing dark silhouettes (white asterisks), hyporefractile compared to the surrounding stroma, speckled with brightly refractile inflammatory cells (thin yellow arrows).
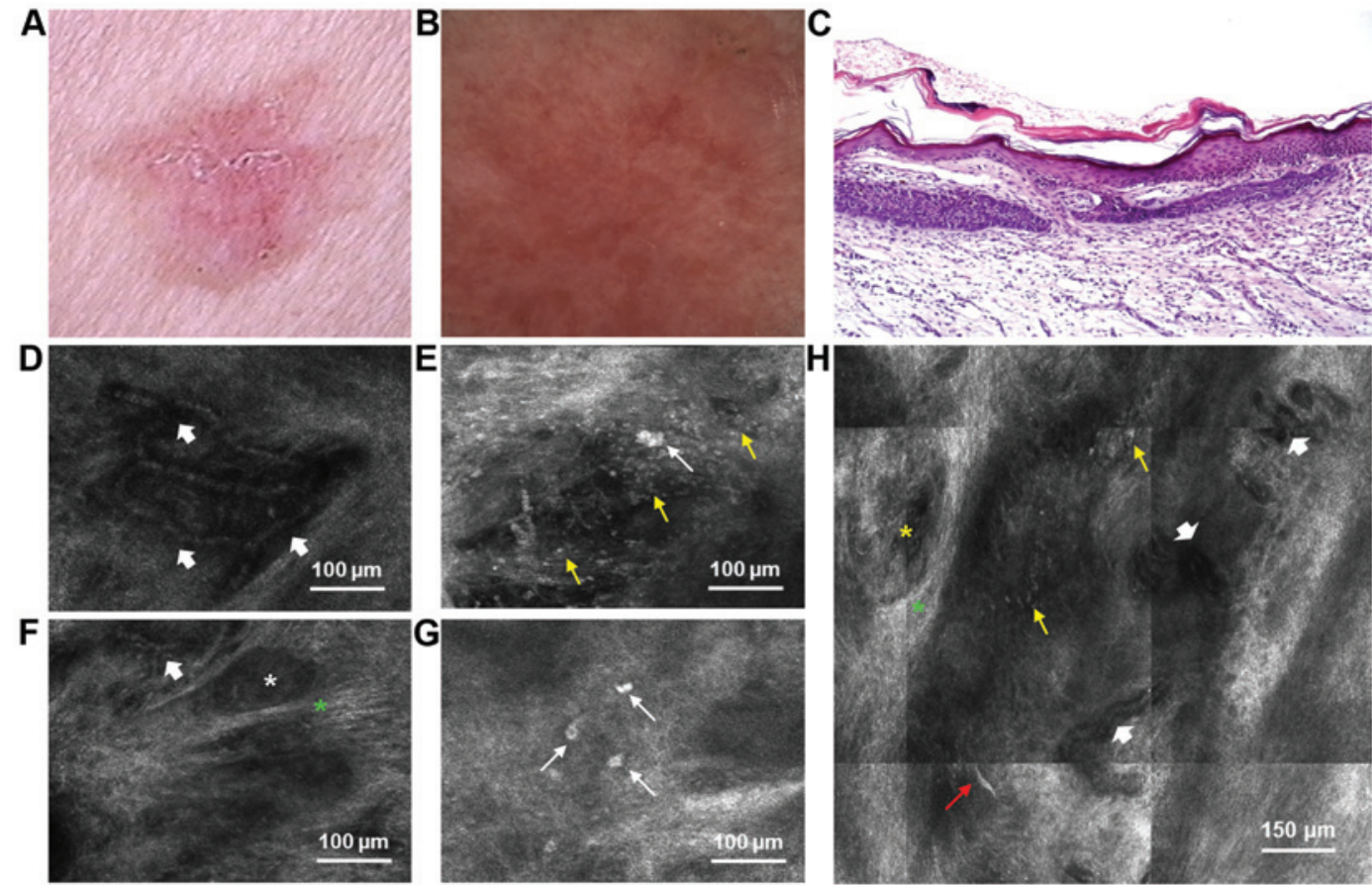

Figure 3. Lesion no. 3. (A) Clinical image of an erythematous, slightly scaly plaque with a stelate shape; (B) dermoscopic image showing pink to red structureless areas, multiple arborizing microvessels, superficial fine telangiectasias and few brown dots; (C) histopathological examination reveals islands of basaloid cells in the papillary dermis, with characteristic peripheral palisading (hematoxylin and eosin staining, x10 magnification); (D) Reflectance confocal microscopy (RCM) image of the dermis revealing tortuous, dilates capillaries (short white arrows); (E) RCM image at the level of upper dermis showing clusters of inflammatory (thin yellow arrows) and plump cells (thin white arrow); (F) RCM image of hyporefractile tumor islands (white asterisk) embedded by bright, fibrous stroma (green asterisk). Dichotomous capillaries can be seen in the upper and lower parts of the image (short white arrow); (G) RCM image of bright, plump cells (thin white arrows) within a tumor mass; (H) RCM image at the level of the upper dermis showing a tumor island (yellow asterisk) that is surrounded by highly-refractile collagen (green asterisk), tortuous, dilated capillaries (short white arrows), clustered inflammatory cells (thin yellow arrows) and a highly-refractile dendritic structure (thin red arrow). 
Table I. RCM morphological features and their dermoscopic and histopathological correlations identified in lesions from patients with sBCC.

\begin{tabular}{ccccc}
\hline RCM feature & Lesion & Lesion & Lesion & Dermoscopic \\
no. 1 & no. 2 & no. 3 & correlation & $\begin{array}{c}\text { Histopathological } \\
\text { correlation }\end{array}$
\end{tabular}

\begin{tabular}{|c|c|c|c|c|c|}
\hline Altered honeycomb pattern & + & + & + & - & $\begin{array}{l}\text { Pleomorphic keratinocytes with atypical } \\
\text { nuclei and scant cytoplasm }\end{array}$ \\
\hline $\begin{array}{l}\text { Nuclear streaming in the } \\
\text { epidermis }\end{array}$ & + & + & + & - & - \\
\hline $\begin{array}{l}\text { Small and round to oval } \\
\text { superficial refractile tumor } \\
\text { islands }\end{array}$ & + & + & + & $\begin{array}{l}\text { Pink-white } \\
\text { structureless areas }\end{array}$ & $\begin{array}{l}\text { Flattening of the rete ridge pattern and } \\
\text { decrease/absence of melanin due to } \\
\text { islands of basaloid tumor cells }\end{array}$ \\
\hline Dark silhouettes & - & + & + & & \\
\hline \multirow[t]{2}{*}{$\begin{array}{l}\text { Highly refractile dots, dendritic } \\
\text { and granular structures }\end{array}$} & + & + & + & Brown/blue-gray dots & $\begin{array}{l}\text { Melanophages, melanocytes or melanin } \\
\text { deposition within tumor islands at the } \\
\text { dermoepidermal junction/papilllary dermis } \\
\text { and/or located in the dermis }\end{array}$ \\
\hline & + & + & + & Blue-gray globules & \\
\hline Peripheral palisading of nuclei & + & - & - & - & $\begin{array}{l}\text { Peripheral palisading at the periphery of } \\
\text { tumor islands }\end{array}$ \\
\hline Clefts & + & + & + & - & $\begin{array}{l}\text { Peritumoral cleft-like spaces corresponding } \\
\text { to the peritumoral mucin deposition }\end{array}$ \\
\hline $\begin{array}{l}\text { Highly reflactile peritumoral } \\
\text { fibrosis }\end{array}$ & + & + & + & - & $\begin{array}{l}\text { Thick collagen bundles surrounding } \\
\text { tumor islands }\end{array}$ \\
\hline $\begin{array}{l}\text { Increased vasculature, with } \\
\text { dilated and branched vessels }\end{array}$ & + & + & + & $\begin{array}{l}\text { Pink to red hue of the } \\
\text { structureless areas. } \\
\text { Short superficial } \\
\text { telangiectasias. } \\
\text { Arborising microvessels }\end{array}$ & $\begin{array}{l}\text { Abundant blood capillaries, often with } \\
\text { dilated and tortuous caliber, adjacent to } \\
\text { basaloid islands }\end{array}$ \\
\hline Inflammatory cells & - & + & + & - & $\begin{array}{l}\text { Peritumoral inflammatory infiltrate } \\
\text { consisting of lymphocytes, histiocytes }\end{array}$ \\
\hline Plump cells & + & + & + & Gray dots & Melanophages/inflammatory cells \\
\hline $\begin{array}{l}\text { Bright dendritic shaped } \\
\text { structures }\end{array}$ & - & - & + & - & Benign melanocytes, Langerhans cells \\
\hline Erosions & - & + & - & Erosions & $\begin{array}{l}\text { Small loss of substance covered by } \\
\text { hematic and lymphocytic exudates }\end{array}$ \\
\hline Dermal solar elastosis & + & + & + & - & $\begin{array}{l}\text { Thickened, irregular basofilic collagen } \\
\text { fibers in the dermis }\end{array}$ \\
\hline
\end{tabular}

RCM, reflectance confocal microscopy; sBCC, superficial basal cell carcinoma.

oval cells were present within and at the periphery of the tumor islands (Fig. 3E and G). Highly refractile projections, possibly belonging to dendritic cells could also be observed (Fig. 3H). Histopathological analysis revealed islands of basaloid cells in the papillary dermis, with characteristic peripheral palisading, thus confirming the diagnosis of sBCC (Fig. 3C).

\section{Discussion}

The presented instructive case reports demonstrate the usefulness of dermoscopy and RCM for the rapid, in vivo, non-invasive evaluation of sBCC, suggesting that a combination of these two diagnostic methods may significantly reduce the number of necessary skin biopsies. This is of great importance to everyday dermatological practice, considering the invasiveness associated with biopsies, high costs and elapsed time until receiving the histopathological report and the recommended non-surgical treatments for sBCC.

Dermoscopy is nowadays indispensable for the dermatologist in the non-invasive, in vivo diagnosis of $\operatorname{BCC}(5,6)$ and there is a growing number of studies that describe relevant dermoscopic criteria for differentiating between BCC subtypes $(4,7)$. Shiny white to red areas and short fine telangiectasias are considered the dermoscopic hallmarks of sBCC, with multiple small erosions as an additional dermoscopic clue for the diagnosis $(8,9)$. Moreover, a group of investigators proposed a dermoscopic algo- 
rithm that could discriminate $\mathrm{sBCC}$ from other BCC subtypes with a sensitivity of $81.9 \%$ and a specificity of $81.1 \%$ (7). In the cases presented herein, dermoscopic images were captured at 10 -fold magnification prior to RCM imaging. Dermoscopic criteria for sBCC were identified, as previously reported (4,7-9), with the presence of pink structureless areas, short superficial telangiectasias and arborizing microvessels in all 3 lesions.

RCM is currently the most promising non-invasive imaging technique for the quasi-microscopic characterization of skin tumors located between the stratum corneum and the superficial reticular dermis (10). RCM diagnostic features of BCC have been described (10-12), including its superficial type $(4,13)$. In particular, the presence of epidermal streaming, cords connected to the epidermis $(4)$ or small $(<300 \mu \mathrm{m})$, round tumor islands connected to the base of the epidermis and solar elastosis (13) were shown to differentiate sBCC from other BCC subtypes. In the cases reported herein, RCM imaging was performed prior to histopathological examination by means of a commercially available reflectance mode confocal laser scanning microscope (Vivascope 1500; Lucid Inc.). A detailed description of the technique and acquisition method has been reported elsewhere $(14,15)$. The procedures were in accordance with the ethical standards of the institutional committee on human experimentation and with the 1975 Helsinki Declaration as revised in 1983.

Our results confirm the presence of previously identified $\mathrm{RCM}$ criteria for $\mathrm{sBCC}$, demonstrating a good correlation with certain dermoscopic and histopathological findings (Table I). Moreover, some RCM morphologic features did not have a dermoscopic correspondent, emphasizing the complementarity of these two in vivo, non-invasive diagnostic techniques. Dark silhouettes, peritumoral clefts and solar elastosis are only some of these features. Dark silhouettes correspond to hypopigmented tumor islands and are visible as dark footprint-like shadows outlined by bright collagen (4). Clefts are defined as peritumoral, dark hyporefractile spaces (16) and dermal solar elastosis refers to the presence of highly refractile, coarse and thick collagen fibres in the dermis (17). Furthermore, some identified RCM characteristics could not be associated with specific histological patterns, suggesting that RCM may provide additional morphologic information, useful for the non-invasive characterization of BCC (4). One such example is the streaming of the epidermis, considered one of the most significant parameters for BCC diagnosis (11) and it refers to the elongated monomorphic keratinocyte nuclei that are oriented along the same axis, mostly observed in the spinous layer (11). In addition to that, plump cells defined as bright, oval or star-shaped cells, larger than inflammatory cells, are usually identified as melanophages or a specific type of inflammatory cells; however, there are limited immunohistochemical studies to differentiate between them (13).

In addition, this novel technology may be a diagnostic guide in defining the margins of the lesion prior to laser ablation (1) or surgical excision (18). Moreover, in contrast to invasive techniques, RCM also allows for the dynamic evaluation of skin vasculature in vivo, in real-time $(19,20)$ and it offers the advantage of performing determinations to monitor tumor response to non-invasive treatment for sBCC (10).

In addition, RCM is a step forward in the evaluation of skin tumors at the cellular level and may contribute to unveiling the mechanisms of cancer development which are of overwhelming complexity $(21,22)$.

In conclusion, in this study, we demonstrate that RCM, combined with dermoscopy allows for the rapid, in vivo, non-invasive micromorphological evaluation of sBCC, that may replace the histopathological examination in this subtype of BCC. However, further larger scale studies are warranted to further validate our findings if the implementation of RCM in daily dermatological practice is to become a reality.

\section{Acknowledgements}

This study was supported by the Sectorial Operational Programme Human Resources Development (SOPHRD), financed by the European Social Fund and the Romanian Government under the contract no. POSDRU 141531/2014, grant no. PN-II-PT-PCCA-2013-4-1407 (project 190/2014) financed by Executive Agency for Higher Education, Research, Development and Innovation and Young Researchers grant no. 33891/2014 financed by 'Carol Davila' University of Medicine and Pharmacy, Bucharest, Romania.

\section{References}

1. Chen CS, Sierra H, Cordova M and Rajadhyaksha M: Confocal microscopy-guided laser ablation for superficial and early nodular Basal cell carcinoma: A promising surgical alternative for superficial skin cancers. JAMA Dermatol 150: 994-998, 2014.

2. Astarci HM, Gurbuz GA, Sengul D, Hucumenoglu S, Kocer U and Ustun H: Significance of androgen receptor and CD10 expression in cutaneous basal cell carcinoma and trichoepithelioma. Oncol Lett 10: 3466-3470, 2015.

3. Kim SA, Lee KS and Cho JW: Photodynamic therapy combined with cryotherapy for the treatment of nodular basal cell carcinoma. Oncol Lett 6: 939-941, 2013.

4. Longo C, Lallas A, Kyrgidis A, Rabinovitz H, Moscarella E, Ciardo S, Zalaudek I, Oliviero M, Losi A, Gonzalez S, et al: Classifying distinct basal cell carcinoma subtype by means of dermatoscopy and reflectance confocal microscopy. J Am Acad Dermatol 71: 716-724.e1, 2014.

5. Altamura D, Menzies SW, Argenziano G, Zalaudek I, Soyer HP, Sera F, Avramidis M, DeAmbrosis K, Fargnoli MC and Peris K: Dermatoscopy of basal cell carcinoma: Morphologic variability of global and local features and accuracy of diagnosis. J Am Acad Dermatol 62: 67-75, 2010.

6. Micantonio T, Gulia A, Altobelli E, Di Cesare A, Fidanza R, Riitano A, Fargnoli MC and Peris K: Vascular patterns in basal cell carcinoma. J Eur Acad Dermatol Venereol 25: 358-361, 2011.

7. Lallas A, Tzellos T, Kyrgidis A, Apalla Z, Zalaudek I, Karatolias A, Ferrara G, Piana S, Longo C, Moscarella E, et al: Accuracy of dermoscopic criteria for discriminating superficial from other subtypes of basal cell carcinoma. J Am Acad Dermatol 70: 303-311, 2014.

8. Scalvenzi M, Lembo S, Francia MG and Balato A: Dermoscopic patterns of superficial basal cell carcinoma. Int J Dermatol 47: 1015-1018, 2008.

9. Giacomel J and Zalaudek I: Dermoscopy of superficial basal cell carcinoma. Dermatol Surg 31: 1710-1713, 2005.

10. González S, Sánchez V, González-Rodríguez A, Parrado C and Ullrich M: Confocal microscopy patterns in nonmelanoma skin cancer and clinical applications. Actas Dermosifiliogr 105: 446-458, 2014

11. Nori S, Rius-Díaz F, Cuevas J, Goldgeier M, Jaen P, Torres A and González S: Sensitivity and specificity of reflectance-mode confocal microscopy for in vivo diagnosis of basal cell carcinoma: A multicenter study. J Am Acad Dermatol 51: 923-930, 2004.

12. Căruntu C, Boda D, Guţu DE and Căruntu A: In vivo reflectance confocal microscopy of basal cell carcinoma with cystic degeneration. Rom J Morphol Embryol 55: 1437-1441, 2014. 
13. Peppelman M, Wolberink EA, Blokx WA, van de Kerkhof PC van Erp PE and Gerritsen MJ: In vivo diagnosis of basal cell carcinoma subtype by reflectance confocal microscopy. Dermatology 227: 255-262, 2013.

14. Calzavara-Pinton P, Longo C, Venturini M, Sala R and Pellacani G: Reflectance confocal microscopy for in vivo skin imaging. Photochem Photobiol 84: 1421-1430, 2008.

15. González S, Swindells K, Rajadhyaksha M and Torres A: Changing paradigms in dermatology: Confocal microscopy in clinical and surgical dermatology. Clin Dermatol 21: 359-369, 2003.

16. Wolberink EA, Pasch MC, Zeiler M, van Erp PE and Gerritsen MJ: High discordance between punch biopsy and excision in establishing basal cell carcinoma subtype: Analysis of 500 cases. J Eur Acad Dermatol Venereol 27 : 985-989, 2013.

17. Longo C, Casari A, Beretti F, Cesinaro AM and Pellacani G: Skin aging: In vivo microscopic assessment of epidermal and dermal changes by means of confocal microscopy. J Am Acad Dermatol 68: e73-e82, 2013.
18. Pan ZY, Lin JR, Cheng TT, Wu JQ and Wu WY: In vivo reflectance confocal microscopy of Basal cell carcinoma: Feasibility of preoperative mapping of cancer margins. Dermatol Surg 38: 1945-1950, 2012.

19. Căruntu C, Boda D, Căruntu A, Rotaru M, Baderca F and Zurac S: In vivo imaging techniques for psoriatic lesions. Rom J Morphol Embryol 55 (Suppl 3): 1191-1196, 2014.

20. Căruntu C and Boda D: Evaluation through in vivo reflectance confocal microscopy of the cutaneous neurogenic inflammatory reaction induced by capsaicin in human subjects. J Biomed Opt 17: 085003, 2012.

21. Spandidos DA: A unified theory for the development of cancer. Biosci Rep 6: 691-708, 1986.

22. Spandidos DA: The cancer story. Cancer Biol Ther 3: 1184-1186, 2004. 